\title{
Testing the potential of a virtual reality neurorehabilitation system during performance of observation, imagery and imitation of motor actions recorded by wireless functional near- infrared spectroscopy (fNIRS)
}

Lisa Holper ${ }^{1,2^{*}}$, Thomas Muehlemann ${ }^{1,3}$, Felix Scholkmann ${ }^{1}$, Kynan Eng $^{2}$, Daniel Kiper ${ }^{2}$, Martin Wolf ${ }^{1}$

\begin{abstract}
Background: Several neurorehabilitation strategies have been introduced over the last decade based on the socalled simulation hypothesis. This hypothesis states that a neural network located in primary and secondary motor areas is activated not only during overt motor execution, but also during observation or imagery of the same motor action. Based on this hypothesis, we investigated the combination of a virtual reality (VR) based neurorehabilitation system together with a wireless functional near infrared spectroscopy (fNIRS) instrument. This combination is particularly appealing from a rehabilitation perspective as it may allow minimally constrained monitoring during neurorehabilitative training.
\end{abstract}

Methods: fNIRS was applied over F3 of healthy subjects during task performance in a virtual reality (VR) environment: 1) 'unilateral' group ( $N=15)$, contralateral recording during observation, motor imagery, observation \& motor imagery, and imitation of a grasping task performed by a virtual limb (first-person perspective view) using the right hand; 2) 'bilateral' group ( $(N=8)$, bilateral recording during observation and imitation of the same task using the right and left hand alternately.

Results: In the unilateral group, significant within-condition oxy-hemoglobin concentration $\Delta\left[\mathrm{O}_{2} \mathrm{Hb}\right]$ changes (mean $\pm \mathrm{SD} \mu \mathrm{mol} / \mathrm{l})$ were found for motor imagery $(0.0868 \pm 0.5201 \mu \mathrm{mol} / \mathrm{l})$ and imitation $(0.1715 \pm 0.4567 \mu \mathrm{mol} / \mathrm{l})$. In addition, the bilateral group showed a significant within-condition $\Delta\left[\mathrm{O}_{2} \mathrm{Hb}\right]$ change for observation $(0.0924 \pm$ $0.3369 \mu \mathrm{mol} / \mathrm{l})$ as well as between-conditions with lower $\Delta\left[\mathrm{O}_{2} \mathrm{Hb}\right]$ amplitudes during observation compared to imitation, especially in the ipsilateral hemisphere $(p<0.001)$. Further, in the bilateral group, imitation using the non-dominant (left) hand resulted in larger $\Delta\left[\mathrm{O}_{2} \mathrm{Hb}\right]$ changes in both the ipsi- and contralateral hemispheres as compared to using the dominant (right) hand.

Conclusions: This study shows that our combined VR-fNIRS based neurorehabilitation system can activate the action-observation system as described by the simulation hypothesis during performance of observation, motor imagery and imitation of hand actions elicited by a VR environment. Further, in accordance with previous studies, the findings of this study revealed that both inter-subject variability and handedness need to be taken into account when recording in untrained subjects. These findings are of relevance for demonstrating the potential of the VR-fNIRS instrument in neurofeedback applications.

\footnotetext{
* Correspondence: holper@ini.phys.ethz.ch

'Biomedical Optics Research Laboratory (BORL), Division of Neonatology, Department of Obstetrics and Gynecology, University Hospital Zurich,

Frauenklinikstrasse 10, 8091 Zurich, Switzerland

Full list of author information is available at the end of the article
} 


\section{Introduction}

Neurorehabilitation based on the simulation hypothesis

Over the last decades, promising strategies in neurorehabilitation, e.g. following cerebral stroke [1-3], have been introduced based on the so-called simulation hypothesis $[4,5]$. The hypothesis suggests that the neural networks of a action-observation system located in the primary motor cortex (M1) and secondary motor areas, such as premotor cortex (PMC), supplementary motor area (SMA) and the parietal cortices, are not only activated during overt motor execution, but also during observation or imagery of the same motor action [6]. These networks are activated when individuals learn motor actions via execution (as in traditional motor learning), imitation, observation (as in observational learning) and motor imagery. Activation of these brain areas following observation or motor imagery may thereby facilitate subsequent movement execution by directly matching the observed or imagined action onto the internal simulation of that action [7]. It is therefore believed that this multi-sensory action-observation system enables individuals to (re)learn impaired motor functions through the activation of these internal action-related representations [8].

We have integrated this knowledge in a novel neurorehabilitative treatment system, based on motor and imagery performance in a virtual reality (VR) environment [9]: the system consists of a VR environment containing virtual representations of the patient's own arms and hands, which are displayed on a large screen and controlled by the patient wearing arm position trackers and data gloves. To activate the action-observation system, patients can train impaired upper limb function by playing interactive games in which they have to perform or imagine specific upper limb movements to interact with the VR environment. By adjustably mapping the movements of both the paretic and healthy limb onto the virtual limbs, the system offers individual training of upper limb motor function even in patients with little arm or hand movement ability.

\section{Functional near-infrared spectroscopy}

To monitor the VR system's effects on brain activation, we chose functional near-infrared spectroscopy (fNIRS). fNIRS is a non-invasive technique based on neurovascular coupling, which exploits the effect of metabolic activity due to neural processing on the oxygenation of cerebral tissue. Utilizing this tight coupling between neuronal activity and localized cerebral blood flow, fNIRS measures hemodynamic changes associated with cortical activation [10]. Optical NIR technology has been shown to be a reliable tool for functional neuroimaging of the human brain [11]. Although NIR technologies feature lower spatial resolution and are only able to image cortical tissue while not providing deeper tissue interrogation as compared to traditional neuroimaging methods such as functional magnetic resonance imaging (fMRI), they offer the advantage of portable systems and, in theory, insensitivity to electromagnetic fields and ferromagnetic materials. In this study a novel miniaturized wireless fNIRS instrument was used [12]. This wireless and portable NIRS technology does not require the subject's body or head to be restrained, and therefore represents an optimal brain monitoring tool for our purpose to record from subjects performing movements in a VR environment. It is thought that this wireless fNIRS technology could overcome some of the limitations inherent to traditional neuroimaging methods.

While the action-observation system described above has been widely investigated using traditional neuroimaging methods [13-15], so far there are only a few studies using NIRS based techniques [16-19]. Further studies have shown fNIRS to be a reliable tool to measure brain oxygenation related to motor imagery performance [20-27], confirming the well-known cortical areas located in primary and secondary motor areas.

The focus of the present study was to obtain evidence of the VR system's efficacy in neurorehabilitation by evaluating its effects on brain activation. In particular, we aimed 1) to provide evidence, that our VR system is able to elicit the action-observation system and 2) to draw conclusions for the system's further application in neurorehabilitative treatment. We hypothesized that the observation, imagery and imitation of a hand motor task in an interactive VR environment enhances the related cortical oxygenation changes of the action-observation system as measured by fNIRS. The long-term aim is to implement the data obtained in the development of a VR-fNIRS based brain computer interfaces (BCIs). Such a VR-fNIRS based BCI could enhance patients' motivation by providing real-time neurofeedback thereby allowing therapists to record pre-post treatment progress assessing training-induced oxygenation changes.

\section{Materials and methods Subjects}

Right-handed subjects were recruited via advertisements at the University of Zurich and ETH Zurich. Exclusion criteria were any history of visual, neurological or psychiatric disorders or any current medication. All subjects gave informed consent. All subjects had normal or corrected-to-normal vision. The study was approved by the ethics committee of the Canton of Zurich and was in accordance with the latest version of the Helsinki declaration. 


\section{Experimental protocol}

Prior to recording, subjects completed the Edinburgh Handedness Inventory (EHI) [28] assessing hand dominance to exclude left-handed subjects. The right-handed subjects were assigned to one of two groups: either to the 'unilateral' group $(\mathrm{N}=15)$ or to the 'bilateral' group $(\mathrm{N}=8)$. Each subject in either group participated in one experimental session. However, bilateral wireless NIRS measurements are more demanding with respect to the instrumentation: two devices are needed instead of one and they must be time-synchronized.

All experiments were conducted in a quiet room. Subjects sat in front of a custom made VR table-system with a computer screen $(94 \mathrm{~cm}$ diagonal) to display the VR environment [9]. The subjects were asked to place their hands on the table with the palms facing downwards, and faced the monitor at a distance of approximately $70 \mathrm{~cm}$. The image on the monitor showed a virtual arm in the same orientation and relative position as the real arms, resting on a flat surface representing the table. The close correspondence between the virtual and real arms in terms of position and relative (firstperson) orientation was designed to optimally stimulate the patient to imagine the virtual arms as their own during the experimental session.

\section{Unilateral group}

In the subject group 'unilateral', fNIRS was recorded over the left hemisphere while the subject performed the VR tasks under four conditions:

- 'Observation $(\mathrm{O})$ ': subjects passively watched a VR video which displayed a right upper limb with the hand repeatedly grasping an incoming ball (13 actions, approx. $0.86 \mathrm{~Hz}$ ) (Figure 1).

- 'Observation \& motor imagery (O\&MI)': same as condition $\mathrm{O}$, except that subjects were asked to imagine that the virtual arm was their own.
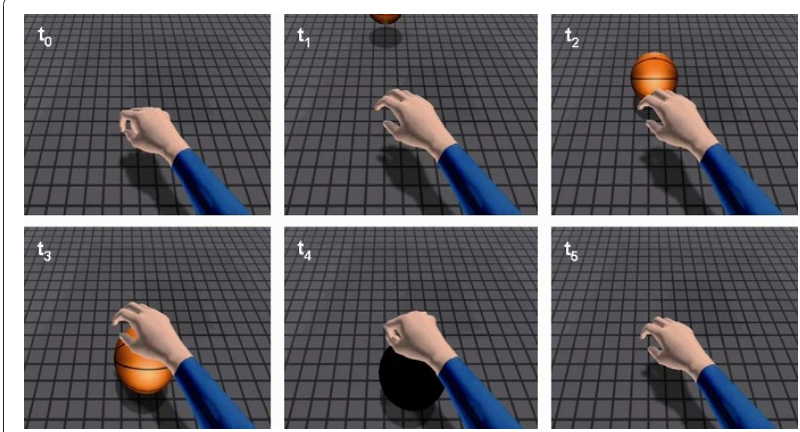

Figure 1 Ball catching task (13 actions in $20 \mathrm{~s}$ ) as shown in the VR video (from top left to bottom right).
- 'Motor imagery (MI)': same as condition O\&MI, but without visual input - subjects had to imagine performing the action.

- 'Imitation (IM)': subjects imitated the hand movements performed in the VR task by the virtual arm while watching the VR video.

The session began with a practice trial (approx. $5 \mathrm{~min}$ ) to allow subjects to become familiar with the tasks. After the practice trial, all subjects first performed condition $\mathrm{O}$ followed by a randomized presentation of conditions O\&MI, MI and IM (Easy Randomizer, Version 4.1. [29]). Subjects were reminded to perform the executed or imagined movements with the same frequency as shown in the video (approx. $0.86 \mathrm{~Hz}$ ). Each condition lasted $530 \mathrm{~s}$ ( $8 \mathrm{~min} 50 \mathrm{~s})$ consisting of 10 trials each comprising an initial rest period (30 s), followed by 10 stimulation periods (20 s) alternated with rest periods (30 s) (Figure 2). The total number of trials per subject was 40; the total duration of the experiment was approx. 35 min per subject. We chose these irregular periodic alternations of $20 \mathrm{~s}$ stimulation and $30 \mathrm{~s}$ rest periods to avoid the induction of synchronization of the sequence of the motor stimulation/rest periods in the motor stimulation protocol with systemic rhythms such as heartbeat, respiration and heart rate fluctuations.

\section{Bilateral group}

The subject group 'bilateral' had the same VR task as the group 'unilateral', but was recorded bilaterally. This group was included to test for a lateralized distribution of oxygenation patterns for the ipsi- and contralateral side, as seen in related studies [30-33]. We hypothesized that, on the one side, the hemisphere contralateral to the hand performing the task would show larger $\left[\mathrm{O}_{2} \mathrm{Hb}\right]$ changes as compared to the ipsilateral hemisphere. The detection of larger $\left[\mathrm{O}_{2} \mathrm{Hb}\right]$ changes over the hemisphere contralateral would provide evidence that we were indeed recording from the correct position, i.e. covering motor-related cortical areas. Conditions $\mathrm{O}$ and $\mathrm{MI}$ were chosen as we assumed that these conditions would elicit the smallest oxygenation changes, both unilaterally and bilaterally. Therefore conditions O\&MI and MI were dropped as we assumed that these conditions would follow a similar pattern to the other conditions.

- 'Observation right (O_R)': Same as condition O in the unilateral group.

- 'Observation left (O_L)': Same as condition O_R, except that a left hand was shown in the VR video.

- 'Imitation right (IM_R)': Same as condition IM in the unilateral group.

- 'Imitation left (IM_L)': Same as condition IM_R, except that a left hand was shown in the VR video 


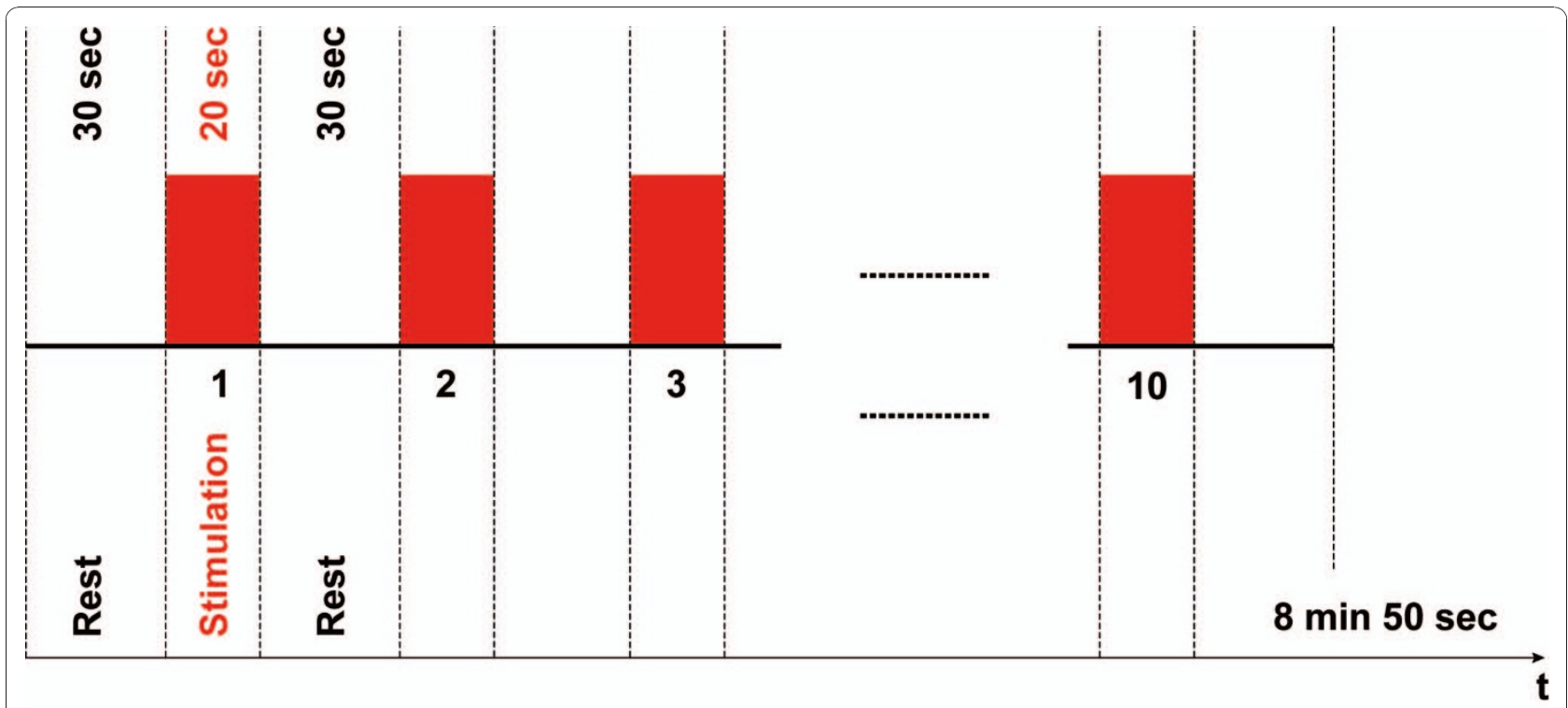

Figure 2 Experimental block design. Each condition consisted of an initial rest period of $30 \mathrm{~s}$, followed by 10 stimulation periods (20 s) alternated with rest periods (30 s). Each condition lasted $530 \mathrm{~s}(8 \mathrm{~min} 50 \mathrm{~s})$; the total duration of the experiment was approx. 35 min per subject.

and subjects were asked to imitate the movement with their left hand.

After the practice trial, all subjects performed condition O_R or O_L first, which was randomly assigned, followed by condition IM_R or IM_L, which was also randomized (Easy Randomizer, Version 4.1. by [29]). The procedure and timing were the same for both the 'unilateral' and the 'bilateral' groups.

\section{NIRS instrumentation}

The novel miniaturized continuous wave wireless fNIRS sensor has been previously described in detail [12]. The optical and electronic components are mounted onto a four-layer rigid-flexible printed circuit board (PCB) which, in combination with a highly flexible casing made of medical grade silicone, enables the sensor to be aligned to curved body surfaces such as the head. The size of the device is $92 \times 40 \times 22 \mathrm{~mm}$ and weighs $40 \mathrm{~g}$. The optical system comprises four light sources at two different wavelengths $(760 \mathrm{~nm}$ and $870 \mathrm{~nm})$ and four detectors (PIN silicon photodiodes). The distance between light sources and detectors is $25 \mathrm{~mm}$, four light source-detector pairs are linearly arranged every 12.5 $\mathrm{mm}$ and thus cover an area of $37.5 \times 25 \mathrm{~mm}$ (Figure 3). Each light source consists of two pairs of serially connected light emitting diodes (LED) is driven using current control and is time multiplexed with an on-time of $120 \mu$ s per sample and a forward voltage of $4 \mathrm{~V}$ per diode. Although LEDs have a broader emission spectrum than lasers, they have several advantages: they can be applied directly on the body surface without need for lenses or fibers and they are inexpensive. Furthermore, they are harmless for the eye, which is an important advantage in a clinical environment. The power is provided by a rechargeable battery, which allows continuous data acquisition for 180 minutes at full light emission power. The light intensity is sampled at $100 \mathrm{~Hz}$ and the resulting data are transmitted wirelessly to the host computer by Bluetooth. The operating range of the sensor is about $5 \mathrm{~m}$. The wireless sensor has been found to be capable of detecting both localized changes $\left[\mathrm{O}_{2} \mathrm{Hb}\right]$ and $[\mathrm{HHb}]$ in the adult brain and oxygenation changes of muscular tissue [12,34].

For fNIRS recording, the sensor(s) was(were) placed either contralateral (unilateral group) or bilaterally (bilateral group) on the subject's head presumably covering F3 according to the international 10-20 system [35]. With the compact sensor of $37.5 \mathrm{~mm}$ length and $25 \mathrm{~mm}$ width, we assumed that we covered secondary motor areas [36]. Hairs under the sensor(s) were carefully brushed away before fixation; shaving was not required. The sensor was fixed on the subject's head using medical-grade, disposable, self-adhesive bandages (Derma Plast CoFix 40 mm, IVF Hartmann, Neuhausen, Switzerland).

For final data processing, by measuring intensity of NIR light after its transmission trough tissue, it is possible to determine changes over time in the concentration of oxy-hemoglobin $\left(\mathrm{O}_{2} \mathrm{Hb}\right)$ and deoxy-hemoglobin $(\mathrm{HHb})$, which represent the dominant light absorbers for living tissue in the NIR spectral band. By applying 


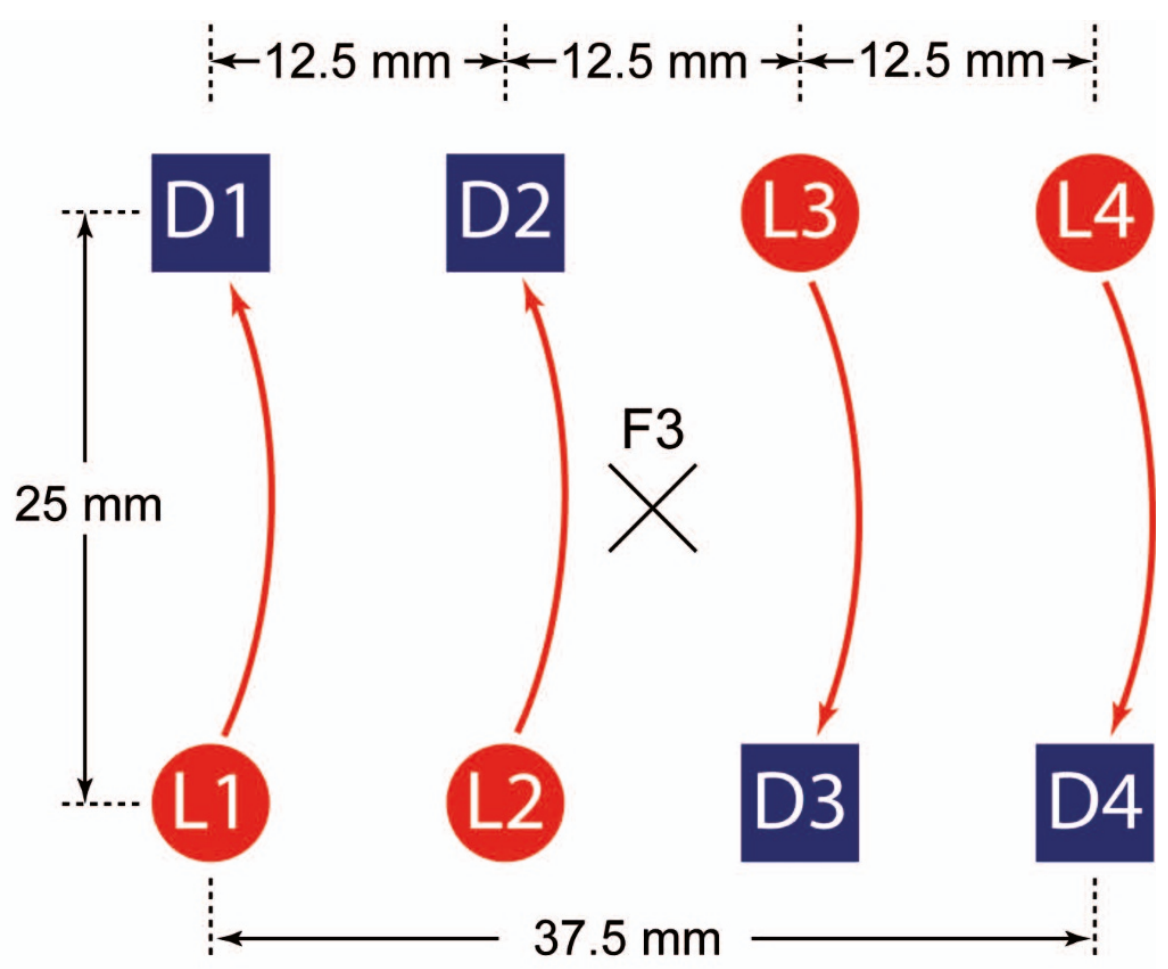

Figure 3 Top-view: schematic of light sources (L1, L2, L3, and L4) and detectors (D1, D2, D3, and D4) on the sensor. The center of the sensor was positioned over position F3 according to the 10-20 system. Four channels were considered for analysis. D1-L1 were in cranial direction, D4-L4 were in caudal direction.

the modified Beer-Lambert law (MBBL), the concentration for $\mathrm{O}_{2} \mathrm{Hb}$ and $\mathrm{HHb}\left(\left[\mathrm{O}_{2} \mathrm{Hb}\right],[\mathrm{HHb}]\right)$ were computed from the measured absorption changes [37,38].

A MATLAB $^{\circ}$ (Version 2008a) program was applied to pre-process the raw light intensity values and to compute $\left[\mathrm{O}_{2} \mathrm{Hb}\right]$ and $[\mathrm{HHb}]$ changes. The measurement files that were acquired during the fNIRS experiment contain the intensity signals of the NIR light, sampled at $100 \mathrm{~Hz}$ for all combinations of light-sources, wavelengths and detectors, as well as the intensity of the ambient light. The program subtracts the ambient light intensities from the NIRS measurement values before low-pass filtering $\left(7^{\text {th }}\right.$ order Chebyshew with $20 \mathrm{~dB}$ attenuation at $5 \mathrm{~Hz}$ ) and decimating the signals to a sampling rate of $10 \mathrm{~Hz}$. Consecutively, the MBLL is used to compute the changes of $\left[\mathrm{O}_{2} \mathrm{Hb}\right]$ and $[\mathrm{HHb}]$ applying differential path lengths factors (DPF) of 6.75 for the $760 \mathrm{~nm}$ and 6.50 for the $870 \mathrm{~nm}$ light-sources [39]. The $\left[\mathrm{O}_{2} \mathrm{Hb}\right]$ and $[\mathrm{HHb}]$ signals acquired with the wireless NIRS signal characteristically drift slightly over time, which can mostly be attributed to thermal effects. Therefore, data was recorded only two minutes after starting the fNIRS sensor, allowing the setup to reach thermal equilibrium. The remaining signal drift [12] was highly linear as assessed by visual inspection and thus their linear least squares approximation was subtracted from $\left[\mathrm{O}_{2} \mathrm{Hb}\right]$ and $[\mathrm{HHb}]$ for drift elimination.

\section{Data Analysis}

Descriptive analysis was calculated for all median signal amplitudes $(\mu \mathrm{mol} / \mathrm{l} \pm \mathrm{SD})$. Each source-detector combination (channel) and each condition was averaged to attempt to provide a detectable signal. The criterion for a detectable signal was the relative value between stimulation and baseline, i.e. increase in $\left[\mathrm{O}_{2} \mathrm{Hb}\right]$ and decrease in $[\mathrm{HHb}]$. At this point those channels that did not show task related oxygenation changes were excluded from further analysis, since it was assumed that those channels did not cover the activated cerebral region at all. For the same reason, subjects that did not display statistically significant changes of the $\left[\mathrm{O}_{2} \mathrm{Hb}\right]$ median for the condition IM (control condition) were excluded as well.

All data were positively tested for Gaussian distribution using the Kolmogorov-Smirnov test. Consecutively, dependant variables for further statistical analysis were derived from the non-excluded $\left[\mathrm{O}_{2} \mathrm{Hb}\right]$ and $[\mathrm{HHb}]$ datasets. Specifically, the median of the last $10 \mathrm{~s}$ of the stimulation periods $\left([\mathrm{HHb}]_{\mathrm{stim}},\left[\mathrm{O}_{2} \mathrm{Hb}\right]_{\mathrm{stim}}\right.$, stimulation amplitudes) and the median of the last $10 \mathrm{~s}$ of the rest periods $\left([\mathrm{HHb}]_{\text {rest }},\left[\mathrm{O}_{2} \mathrm{Hb}\right]_{\text {rest }}\right.$, baselines $)$ were tested in 
the analysis. The median was chosen instead of the mean as it is more robust to outliners that may have statistically unbalanced the analysis in our relatively small subject sample. The statistical significance of the intra-condition differences between $\left([\mathrm{HHb}]_{\text {rest }},\left[\mathrm{O}_{2} \mathrm{Hb}\right]_{\text {rest }}\right)$ and $([\mathrm{HHb}]$ stim, $\left.\left[\mathrm{O}_{2} \mathrm{Hb}\right]_{\text {stim }}\right)$, later referred to as $\Delta[\mathrm{HHb}]$ and $\Delta$ $\left[\mathrm{O}_{2} \mathrm{Hb}\right]$, was analyzed using the paired t-test.

The statistical significance of inter-conditional differences of $\left[\mathrm{O}_{2} \mathrm{Hb}\right]_{\text {stim }}$ and $[\mathrm{HHb}]_{\text {stim }}$ as well as for $[\mathrm{HHb}]$ rest and $\left[\mathrm{O}_{2} \mathrm{Hb}\right]_{\text {rest }}$ were first assessed across all conditions. Then, if a significant difference was found, it was followed by a pair wise comparisons for all possible condition pairs using one-way ANOVA; the alpha-value for significance was set to $\leq 0.05$ and the Bonferroni correction was applied to eliminate the problem of multiple comparisons.

\section{Results}

\section{Behavioral data}

23 healthy subjects were included in the analysis (15 unilateral group, 8 bilateral group, 9 males, mean age 26 years, range 22 - 33 years). Five subjects ( 2 in unilateral group; 3 in bilateral group) were excluded from analysis due to a missing signal in the IM condition. All subjects were right-handed according to the EHI with a mean LQ of 81.9 (range 73 - 100) and a mean deciles level of 6.1 (range 3 - 10).

\section{fNIRS measurements}

\section{Unilateral group}

The mean $\Delta\left[\mathrm{O}_{2} \mathrm{Hb}\right]$ (Table 1 ) was largest in the IM condition, followed by MI, O, and O\&MI. Mean $\Delta[\mathrm{HHb}]$ was largest in condition MI, followed by IM, O\&MI, and $\mathrm{O}$. The data showed a higher degree of inter-subject variability observed for $\Delta\left[\mathrm{O}_{2} \mathrm{Hb}\right]$ compared to $\Delta[\mathrm{HHb}]$ as calculated by the standard deviation (SD) of the oxygenation changes.

Intra-condition analysis of the median changes between $\left[\mathrm{O}_{2} \mathrm{Hb}\right]_{\text {rest }}$ and $\left[\mathrm{O}_{2} \mathrm{Hb}\right]_{\text {stim }}$ using a paired t-test (Table 1) revealed statistical significance in the MI ( $\mathrm{p}=$ $0.049)$ and IM $(p<0.001)$ conditions. No significant differences were detected between $[\mathrm{HHb}]_{\text {rest }}$ and $[\mathrm{HHb}]$ stim. Figure 4 shows an example of a sample subject of the oxygenation changes from rest to stimulation period in each of the four conditions.

Inter-condition analysis of the mean amplitude changes of $\Delta\left[\mathrm{O}_{2} \mathrm{Hb}\right]$ and $\Delta[\mathrm{HHb}]$ between rest and stimulation periods between the four conditions using one-way ANOVA (Table 1, Figure 5) revealed neither a main effect of condition, nor statistical significant between the four conditions.

\section{Bilateral group}

In this group a smaller number of subjects was included, although sufficient to reach statistical significance.
In the left hemisphere, the mean $\Delta\left[\mathrm{O}_{2} \mathrm{Hb}\right]$ (Table 2) were largest in condition IM_L, followed by IM_right, O_R, and O_L. Mean $\Delta[\mathrm{HHb}]$ were largest in condition IM_L, followed by IM_R, O_L and O_R.

On the right hemisphere, mean $\Delta\left[\mathrm{O}_{2} \mathrm{Hb}\right]$ were largest in condition IM_L, followed by IM_R, O_R, and O_L. Mean $\Delta[\mathrm{HHb}]$ were largest in condition IM_L, followed by IM_R, O_L and O_R. As also seen in the unilateral group a relatively high inter-subject variability was observed, as documented by the standard deviation (SD).

Intra-condition analysis (left hemisphere ( $\mathrm{LH}$ ), right hemisphere $(\mathrm{RH})$ ) of the median change between $\left[\mathrm{O}_{2} \mathrm{Hb}\right]$ rest and $\left[\mathrm{O}_{2} \mathrm{Hb}\right]_{\text {stim }}$ using the paired t-test (Table 2) revealed statistical significant differences in conditions O_R (LH p $=0.016$, RH p $=0.006)$, O_L $(\mathrm{LH} \mathrm{p}=0.046$, RH p = 0.025), IM_R (LH p $=0.003$, RH p $<0.001)$ and IM_L $(\mathrm{LH} \mathrm{p}<0.001, \mathrm{RH} \mathrm{p}=0.001)$. Between $[\mathrm{HHb}]_{\text {rest }}$ and $[\mathrm{HHb}]_{\text {stim }}$ statistical significance was observed in condition IM_L (LH p $=0.040$, RH p < 0.001).

Inter-condition analysis of the mean amplitude changes of $\Delta\left[\mathrm{O}_{2} \mathrm{Hb}\right]$ and $\Delta[\mathrm{HHb}]$ between the four conditions using one-way ANOVA (Table 2, Figure 6) revealed a main effect of condition for $\left[\mathrm{O}_{2} \mathrm{Hb}\right](\mathrm{LH} \mathrm{p}=$ 0.028 , RH p $<0.001)$ and for $[\mathrm{HHb}](\mathrm{RH} \mathrm{p}<0.001)$. Statistical significance was found for $\Delta\left[\mathrm{O}_{2} \mathrm{Hb}\right]$ betweenconditions O_R and IM_L $(\mathrm{RH} p<0.001)$, O_L and IM_L (RH p $=<0.001)$ and IM_R and IM_L (RH p < 0.001); analog for $\Delta[\mathrm{HHb}]$ between-conditions $\mathrm{O} \_\mathrm{R}$ and IM_L (RH p < 0.001), O_L and IM_L (RH p $=<0.001)$ and IM_R and IM_L (RH p < 0.001).

In the following discussion we concentrate on the observed $\left[\mathrm{O}_{2} \mathrm{Hb}\right]$ changes, since this parameter shows the relevant significant oxygenation changes, whereas [HHb] did show overall significant levels. This is supported by previous fNIRS work suggesting that interpretations about task-relevant activation increases are usually attributed to the prominent increases in $\left[\mathrm{O}_{2} \mathrm{Hb}\right]$ $[40]$, whereas $[\mathrm{HHb}]$ is often not reported.

\section{Discussion}

\section{Virtual reality based neurorehabilitation}

Recent experimental evidence suggests that rapid advancement of VR technologies has great potential for the development of novel strategies for sensory-motor training in neurorehabilitation [41]. The combination with our wireless and portable fNIRS brain monitoring technique [12] is particularly appealing from a rehabilitation perspective as it allows therapists and patients unconstraint monitoring while testing and training motor performance $[21,42]$.

In this study we provide evidence for the efficacy of our new VR neurorehabilitation system [9] by evaluating its effects on brain activation. In particular, we show that our VR system is able to elicit the action-observation 
Table 1 Unilateral group

\begin{tabular}{|c|c|c|c|c|}
\hline Unilateral group $[\mathrm{N}=15]$ & Observation & Motor imagery & Observation \& motor imagery & Imitation \\
\hline \multicolumn{5}{|c|}{ left hemisphere (contralateral) ( $\mathrm{mmol} / \mathrm{I} \pm \mathrm{SD}$ ) } \\
\hline Mean $\Delta\left[\mathrm{O}_{2} \mathrm{Hb}\right]$ & $0.0692 \pm 0.4510$ & $0.0868 \pm 0.5201$ & $0.0446 \pm 0.5741$ & $0.1715 \pm 0.4567$ \\
\hline Mean $\Delta[\mathrm{HHb}]$ & $-0.0052 \pm 0.1247$ & $0.0356 \pm 0.2043$ & $-0.0089 \pm 0.2391$ & $0.0212 \pm 0.1685$ \\
\hline \multicolumn{5}{|l|}{ T-test, Cl 95\% } \\
\hline$\left[\mathrm{O}_{2} \mathrm{Hb}\right] \mathrm{p}=$ value & $p=0.154$ & $p=0.049^{*}$ & $p=0.333$ & $p<0.001^{*}$ \\
\hline [HHb] p-value & $p=0.161$ & $p=0.061$ & $p=0.760$ & $p=0.323$ \\
\hline \multicolumn{2}{|c|}{ ANOVA, post-hoc-tests, Bonferroni 0.05} & [HHb] p-value & {$\left[\mathrm{O}_{2} \mathrm{Hb}\right] \mathrm{p}=$ value } & \\
\hline & $\mathrm{O}-\mathrm{Ml}$ & $p=0.387$ & $p=1.000$ & \\
\hline & $\mathrm{O}-\mathrm{O} \& \mathrm{Ml}$ & $p=1.000$ & $p=1.000$ & \\
\hline & $\mathrm{O}-\mathrm{IM}$ & $p=1.000$ & $p=0.509$ & \\
\hline & $\mathrm{Ml}-\mathrm{O} \& \mathrm{Ml}$ & $p=0.265$ & $p=1.000$ & \\
\hline & $\mathrm{MI}-\mathrm{IM}$ & $p=1.000$ & $p=0.934$ & \\
\hline & O\&MI - IM & $p=1.000$ & $p=0.194$ & \\
\hline
\end{tabular}

(Top) Mean signal amplitudes ( $\mu \mathrm{mol} / \mathrm{I} \pm \mathrm{SD}$ ) of channels with significant concentration changes. Separate calculations for increases in [O2Hb], decreases in $[\mathrm{HHb}]$ in response to the four conditions for each group. Numbers were rounded to four decimal places. (Middle) Intra-condition statistical significance of the mean changes between $[\mathrm{O} 2 \mathrm{Hb}]$ rest and $[\mathrm{O} 2 \mathrm{Hb}]$ stim and $[\mathrm{HHb}]$ rest and $[\mathrm{HHb}]$ stim using the paired t-test; confidence interval $(\mathrm{Cl})=95 \%$. (Bottom) Inter-condition statistical significance of mean changes of $\Delta[\mathrm{O} 2 \mathrm{Hb}]$ and $\Delta[\mathrm{HHb}]$ between the four conditions using ANOVA. Shown are post-hoc tests (with Bonferroni correction); significant values $(p \leq 0.05)$ are highlighted by * (observation $=0$, motor imagery $=\mathrm{MI}$, observation \& motor imagery $=0 \&$ MI, imitation $=I M)$

system as described by the simulation hypothesis. Based on these results we aim in the long-term to develop a VR-fNIRS based BCI that providing the possibility of real-time neurofeedback combined with an assessment of training-induced cortical oxygenation changes.

\section{Observation, imagery and imitation}

From the comparisons between stimulation and rest periods, our results confirm the simulation hypothesis in accordance with well-known findings in fMRI and EEG $[3,14,15,43,44]$ and previous fNIRS studies [21-25,45] that have shown that oxygenation changes can be found within the same secondary motor areas during observation, motor imagery and overt motor execution (unilateral and bilateral group, Figure 5 and 6). Although not all of the observed changes reached statistical significance, our results revealed that averaged $\Delta\left[\mathrm{O}_{2} \mathrm{Hb}\right]$ during observation and motor imagery were approximately one-third lower compared to the imitation task. This result is in line with the previous studies mentioned above where both imagery and observation have been reported to elicit consistently lower oxygenation changes.

\section{Inter-subject variability}

We observed a high inter-subject variability in $\Delta\left[\mathrm{O}_{2} \mathrm{Hb}\right]$ in both our samples. General reasons for variability between individuals may be effects of anatomical variance such as thickness of the skull or cerebrospinal fluid layers [46,47]. Another contributing factor might be that our subjects had no prior specific experience in the tasks presented. They were not specifically trained to perform the tasks prior to the experiment (but only received a short practice trial), yet this has been done in a previous fNIRS controlled BCI [24]. Therefore, in our untrained subjects, inter-subject variability in the hemodynamic response patterns might have been higher than it would have been after substantial pre-experimental training. The question of the extent to which a person is able to generate a mental representation of movements is even more relevant in the assessment of individuals following brain injury. Lesions involving specific cortical areas may impair certain imagery abilities [48], such as overall slowing of imagery processes resulting in modified temporal characteristics of motor imagery $[49,50]$.

\section{Bilateral oxygenation}

As observed in previous studies, brain activation in response to executed or imagined actions can differ depending on the hemisphere recorded [51-53]. In general, unimanual tasks show hemispheric asymmetry with predominant activation of the contralateral hemisphere controlling the moving hand, as assessed by fMRI and PET [30-33]. Additionally, ipsilateral activation is both found in M1 and shifted laterally, ventrally, and anteriorly towards PMC for unimanual tasks with respect to that observed during contralateral hand movements [54-60]. Accordingly, we observed ipsi- and contralateral oxygenation changes, both during observation and imitation. 

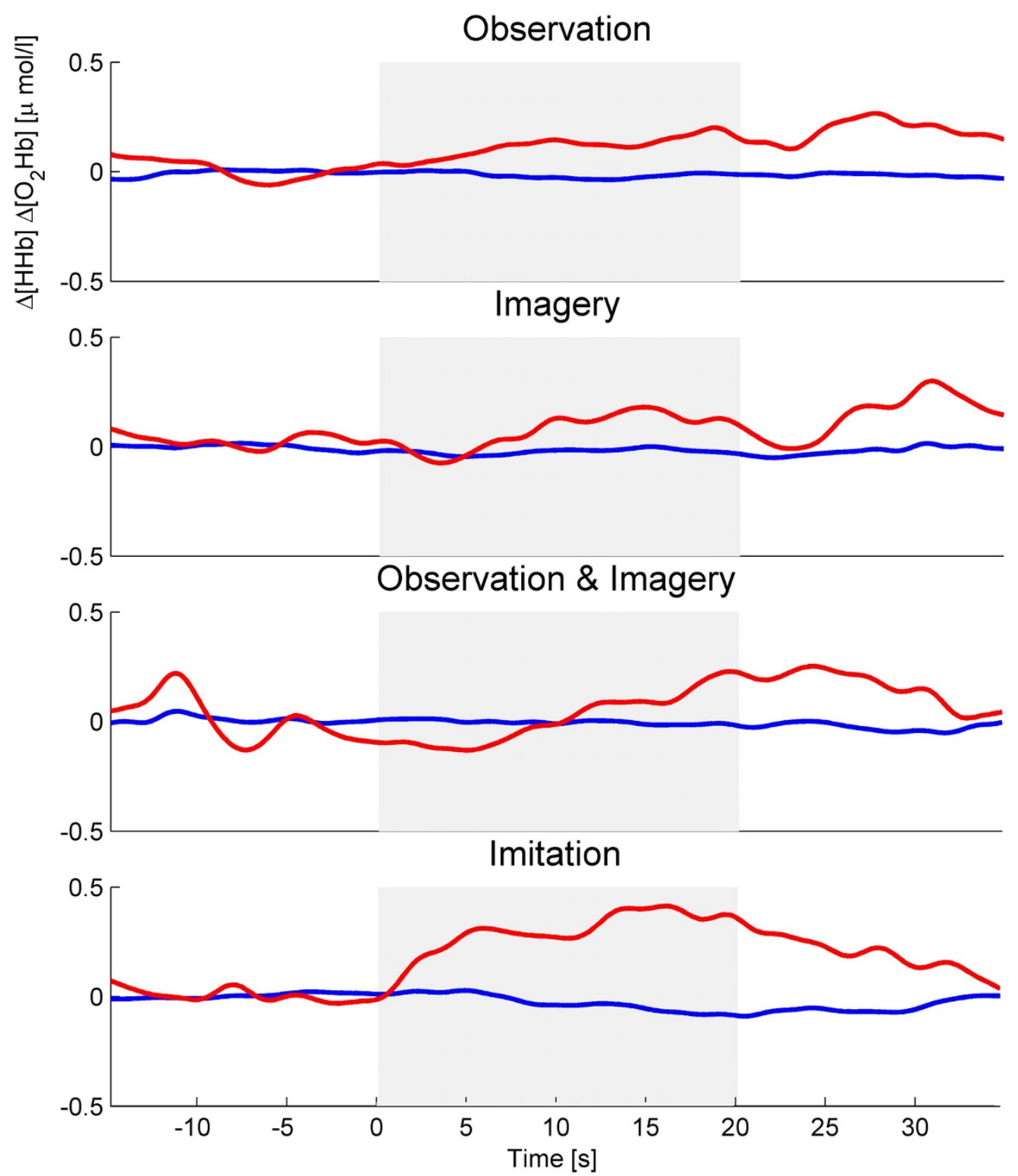

Figure 4 Example of a sample subject of the oxygenation changes $\Delta\left[\mathrm{O}_{2} \mathrm{Hb}\right]$ and $\Delta[\mathrm{HHb}](\mu \mathrm{mol} / \mathrm{l})$ from rest (30 s) to stimulation (20 s) period in each of the four conditions Observation (O), Imagery (MI), Observation \& Imagery (O\&MI) and Imitation (IM). Stimulation onand offset is indicated by the dotted lines.

The difference observed between the unilateral and the bilateral group is concerned about the aspect of handedness. Interestingly, we found that performance during the condition IM_L (imitation with the subject's left non-dominant hand) revealed larger $\Delta\left[\mathrm{O}_{2} \mathrm{Hb}\right]$ in both hemispheres as compared to IM_R (imitation with the subject's right dominant hand) (Figure 6). Further, the $\Delta\left[\mathrm{O}_{2} \mathrm{Hb}\right]$ in the right hemisphere during movement of the subjects' left hand (i.e. the non-dominant, contralateral hand) is considerably larger than that in the left hemisphere during ipsilateral movement. Additionally, in the left hemisphere during ipsilateral movement (non-dominant hand) the $\Delta\left[\mathrm{O}_{2} \mathrm{Hb}\right]$ was larger than that observed during contralateral movement (dominant hand; according to the unilateral group). Figure 5 and 6 reflect these findings showing the observed inter-condition differences in the right hemisphere including lower level $\Delta\left[\mathrm{O}_{2} \mathrm{Hb}\right]$ amplitude during observation as compared to imitation (Figure 6). These findings might be explained by the hand dominance of our right-handed sample. Previous fMRI studies described that non-dominant hand movements appear to require more cortical activity and therefore may result in greater recruitment of ipsi- and contralateral cortical motor areas [61], 


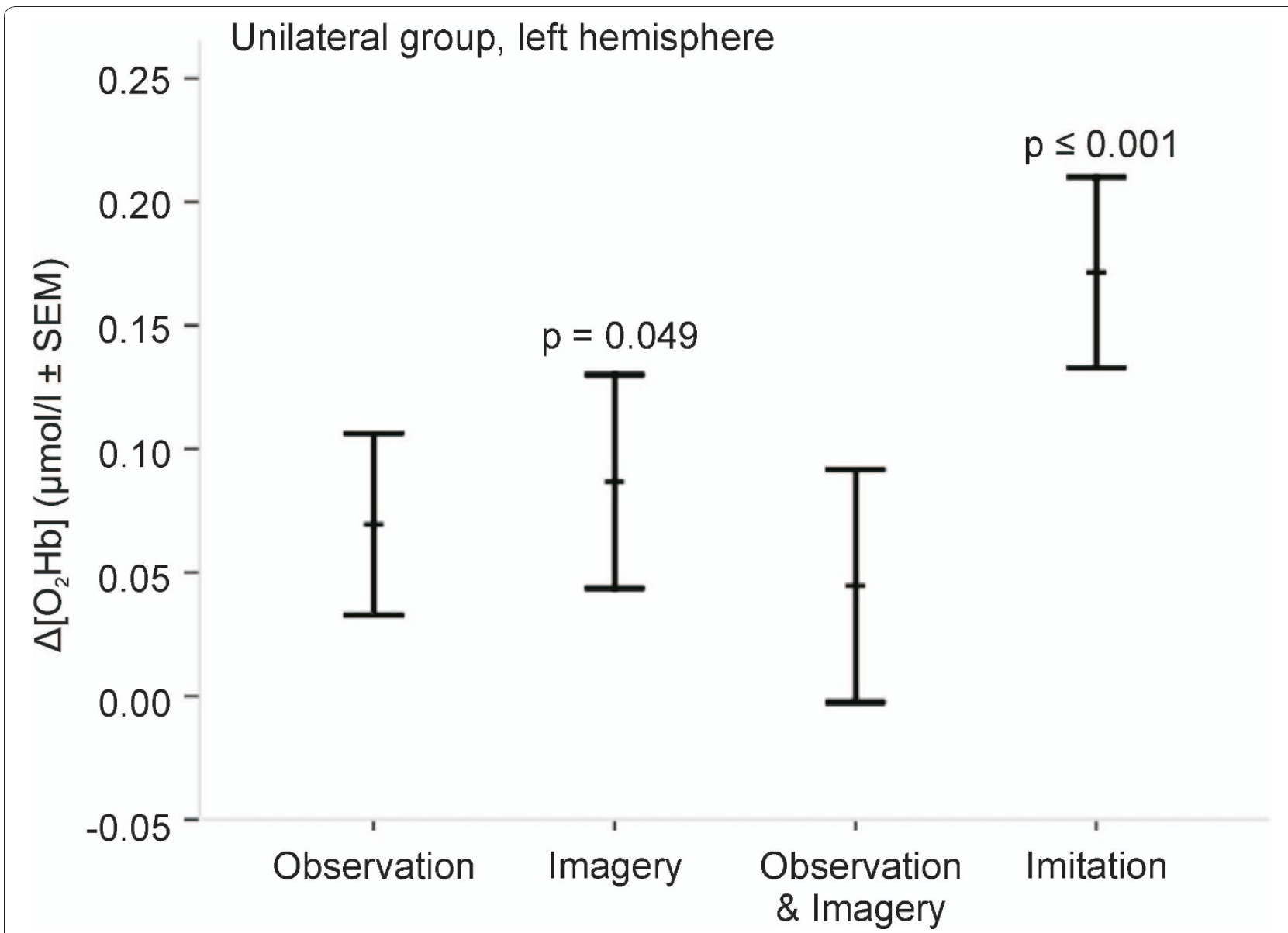

Figure 5 Unilateral group recorded over left hemisphere: diagram of the $\Delta\left[\mathrm{O}_{2} \mathrm{Hb}\right]$ amplitude changes with standard error of the mean (SEM) and statistical significances of paired t-test are shown.

perhaps because they are less 'automatic'. It has been further observed that this ipsilateral activation was most pronounced in pre-central areas (presumably corresponding to secondary motor areas) during both dominant and non-dominant performance [62]. However, further fNIRS studies are needed to confirm whether or not our findings of larger $\Delta\left[\mathrm{O}_{2} \mathrm{Hb}\right]$ during non-dominant performance are in fact caused by the right-handedness of our sample.

\section{Neurorehabilitative potential of combined VR NIRS applications}

Taken together the findings of the uni- and bilateral groups, the results show that our VR system can activate the action-observation system as described by the simulation hypothesis. In particular, 1) the study provides evidence that fNIRS recording does not impede interaction with the VR environment This point is an important precondition for further development of combined VR-fNIRS based applications for use in neurorehabilitation. It increases usability in that it requires a short time to fit fNIRS sensor important for therapy. Further, the results revealed two factors that need to be taken into account when dealing with fNIRS signals aimed to provide a basis for neural interfaces: 2) The inter-subject variability is obvious at the group level and will be even more prominent at he single subject level. The reasons for intersubject variability, i.e. individual experience in motor imagery performance, physiological and anatomical differences, require further assessment. 3) The combined factors of recording side, i.e. uni- or bilateral hemispheres, as well as hand side, i.e. left or right hand used during motor or imagery tasks, need to be taken into account. Our findings may reflect an aspect of handedness in right-handed subjects who may require more cortical activity when using the non-dominant hand. Future studies may include both left-handers and right-handers. Considering these factors may contribute to differentiation of individual oxygenation pattern and permit classification of activation tasks used for neurofeedback or BCI applications. 
Table 2 Bilateral group

\begin{tabular}{|c|c|c|c|c|}
\hline Bilateral group [N = 8] & Observation right & Observation left & Imitation right & Imitation left \\
\hline \multicolumn{5}{|c|}{ Left hemisphere $(\mu \mathrm{mol} / \mathrm{I} \pm \mathrm{SD})$} \\
\hline Mean $\Delta\left[\mathrm{O}_{2} \mathrm{Hb}\right]$ & $0.0924 \pm 0.3369$ & $0.0835 \pm 0.4589$ & $0.1905 \pm 0.5515$ & $0.2712 \pm 0.4424$ \\
\hline Mean $\Delta[\mathrm{HHb}]$ & $-0.0028 \pm 0.1039$ & $-0.0138 \pm 0.1923$ & $0.0206 \pm 0.1569$ & $0.0297 \pm 0.1273$ \\
\hline \multicolumn{5}{|l|}{ T-test, Cl 95\% } \\
\hline$\left[\mathrm{O}_{2} \mathrm{Hb}\right] \mathrm{p}=$ value & $p=0.016^{*}$ & $p=0.046^{*}$ & $p=0.003^{*}$ & $p<0.001^{*}$ \\
\hline [HHb] p-value & $p=0.807$ & $p=0.523$ & $p=0.244$ & $p=0.040^{*}$ \\
\hline \multicolumn{2}{|c|}{ ANOVA, post-hoc-tests, Bonferroni 0.05} & [HHb] p-value & {$\left[\mathrm{O}_{2} \mathrm{Hb}\right] \mathrm{p}=$ value } & \\
\hline & $\mathrm{O}-\mathrm{Ml}$ & $p=1.000$ & $p=1.000$ & \\
\hline & $\mathrm{O}-\mathrm{O} \& \mathrm{Ml}$ & $p=1.000$ & $p=1.000$ & \\
\hline & $\mathrm{O}-\mathrm{IM}$ & $p=1.000$ & $p=0.080$ & \\
\hline & $\mathrm{Ml}-\mathrm{O} \& \mathrm{Ml}$ & $p=0.868$ & $p=0.822$ & \\
\hline & $\mathrm{Ml}-\mathrm{IM}$ & $p=0.393$ & $p=0.056$ & \\
\hline & O\&MI - IM & $p=1.000$ & $p=1.000$ & \\
\hline main effect on condition & & $p=0.222$ & $p=0.028^{*}$ & \\
\hline
\end{tabular}

\section{Right hemisphere $(\mu \mathrm{mol} / \mathrm{l} \pm \mathrm{SD})$}

\begin{tabular}{llll}
\hline Mean $\Delta\left[\mathrm{O}_{2} \mathrm{Hb}\right]$ & $0.1135 \pm 0.3607$ & $0.1091 \pm 0.4261$ & $0.2004 \pm 0.4740$ \\
\hline Mean $\Delta[\mathrm{HHb}]$ & $0.0018 \pm 0.1388$ & $0.0037 \pm 0.1441$ & $0.0163 \pm 0.1325$ \\
\hline
\end{tabular}

\begin{tabular}{|c|c|c|c|c|}
\hline \multicolumn{5}{|l|}{ T-test, Cl 95\% } \\
\hline$\left[\mathrm{O}_{2} \mathrm{Hb}\right] \mathrm{p}=$ value & $p=0.006^{*}$ & $p=0.025^{*}$ & $p<0.001^{*}$ & $p=0.001^{*}$ \\
\hline [HHb] p-value & $p=0.906$ & $p=0.817$ & $p=0.275$ & $p=0.001^{*}$ \\
\hline \multicolumn{2}{|c|}{ ANOVA, post-hoc-tests, Bonferroni 0.05} & [HHb] p-value & \multicolumn{2}{|c|}{$\left[\mathrm{O}_{2} \mathrm{Hb}\right] \mathrm{p}=$ value } \\
\hline & $\mathrm{O}-\mathrm{Ml}$ & $p=1.000$ & \multicolumn{2}{|l|}{$p=1.000$} \\
\hline & $\mathrm{O}-\mathrm{O} \& \mathrm{Ml}$ & $p=1.000$ & \multicolumn{2}{|l|}{$p=1.000$} \\
\hline & $\mathrm{O}-\mathrm{IM}$ & $p<0.001^{*}$ & \multicolumn{2}{|l|}{$p<0.001^{*}$} \\
\hline & $\mathrm{Ml}-\mathrm{O} \& \mathrm{Ml}$ & $p=1.000$ & \multicolumn{2}{|l|}{$p=1.000$} \\
\hline & $\mathrm{Ml}-\mathrm{IM}$ & $p<0.001^{*}$ & \multicolumn{2}{|l|}{$p<0.001^{*}$} \\
\hline & O\&MI - IM & $p<0.001^{*}$ & \multicolumn{2}{|l|}{$p<0.001^{*}$} \\
\hline \multicolumn{2}{|c|}{ main effect on condition } & $p<0.001^{*}$ & \multicolumn{2}{|l|}{$p<0.001^{*}$} \\
\hline
\end{tabular}

(Top) Mean signal amplitudes ( $\mu \mathrm{mol} / \mathrm{I} \pm \mathrm{SD}$ ) of channels with significant concentration changes. Separate calculations for increases in [O2Hb], decreases in [HHb] in response to the four conditions for each group. Numbers were rounded to four decimal places. (Middle) Intra-condition statistical significance of the mean change between $[\mathrm{O} 2 \mathrm{Hb}]$ rest and $[\mathrm{O} 2 \mathrm{Hb}]$ stim and $[\mathrm{HHb}]$ rest and $[\mathrm{HHb}]$ stim using the paired t-test; confidence interval $(\mathrm{Cl})=95 \%$. $(\mathrm{Bottom})$ Inter-condition statistical significance of mean changes of $\Delta[\mathrm{O} 2 \mathrm{Hb}]$ and $\Delta[\mathrm{HHb}]$ between the four conditions using ANOVA. Shown are post-hoc tests (with Bonferroni correction); significant values $(p \leq 0.05)$ are highlighted by * (observation left $=0 \_L$, observation right $=0 \_R$, imitation left $=I M \_L$, imitation right $=I M \_R$ )

\section{Study limitations}

Although the present study revealed interesting results concerning the potential of the new wireless NIRS system, it was subject to some known limitations. We did not record an electromyogram (EMG) in order to exclude the presence of muscular activation during observation and motor imagery. It could be claimed that weak motor activity might have been present during the imagery tasks. However, previous neuroimaging studies suggested that brain signals during imagery of hand motor tasks are not correlated with EMG activity [63]. Another possible limitation is that we referenced the positioning of the NIRS device according to the 10-20 system [35]. However, this positioning may be inaccurate due to inter-subject variability in anatomical head size and shape, and the location on underlying (pre-) motor areas. The location of NIRS recording can therefore generally only be assumed to have correctly covered the preferred areas, i.e. in our case secondary motor areas. 


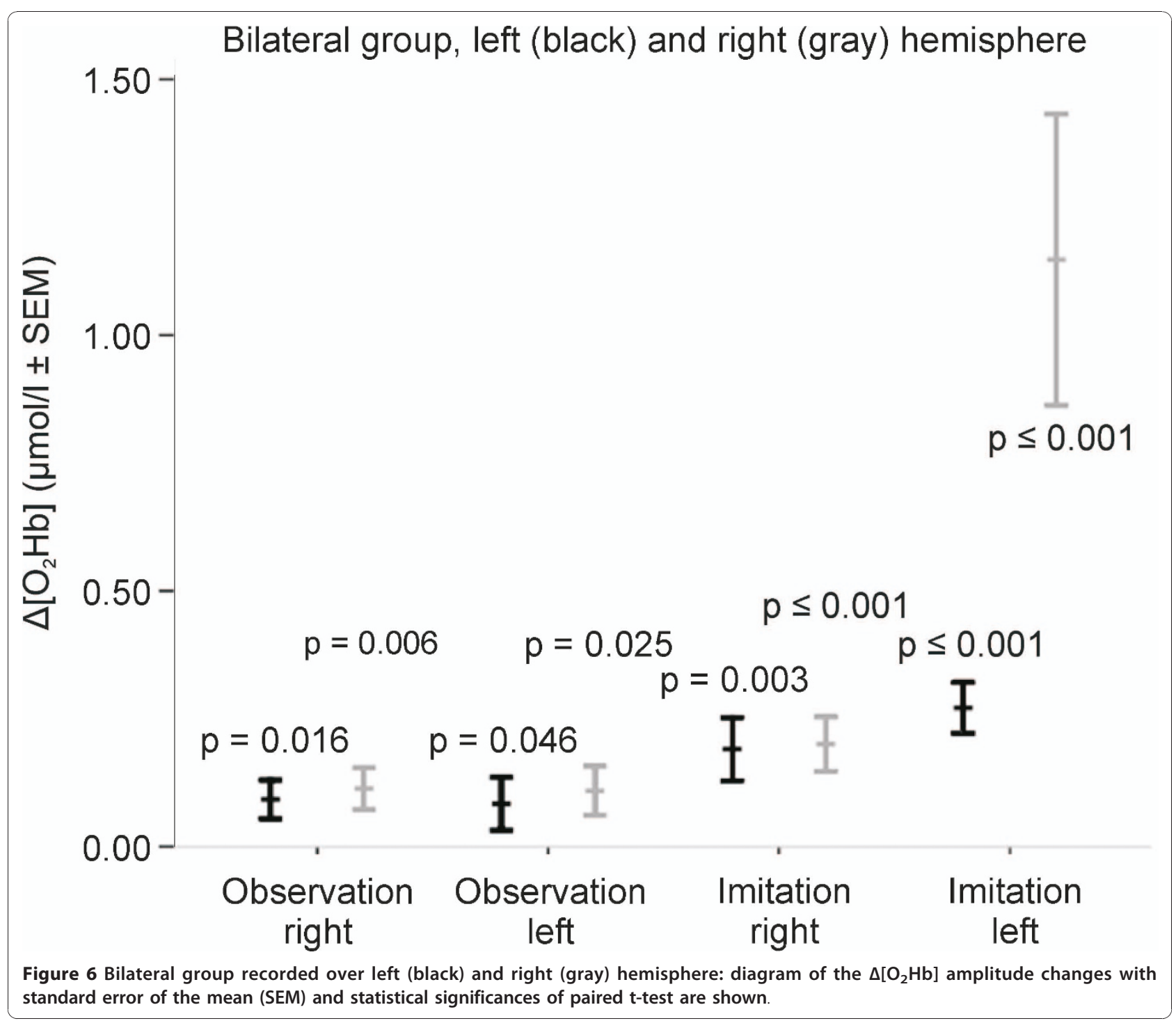

\section{Conclusion}

This study shows that our combined VR-fNIRS based neurorehabilitation system is able to activate the actionobservation system as described by the simulation hypothesis during performance of observation, motor imagery and imitation of hand actions elicited by a VR environment. Further, in accordance with previous studies, the findings of this study revealed that both intersubject variability as well as handedness needs to be taken into account when recording in untrained subjects. In the long term, these findings are of relevance for the VR-fNIRS instrument's potential in neurofeedback applications.

LH conceived of the study, conducted the fNIRS recordings, carried out the statistical analysis, and drafted the manuscript. TM and FS carried out the MATLAB $^{\oplus}$ pre-processing. KE and DK participated in the design of the study. MW participated in the design and coordination of the study. All authors read and approved the final manuscript.

\section{Declaration of competing interests}

The authors declare that they have no competing interests.

\section{Acknowledgements}

The authors thank all participants for their assistance in carrying out this research and the Swiss Society for Neuroscience (SSN), the International Brain Research Organization (IBRO), the Swiss National Research Foundation and the Stiftung für wissenschaftliche Forschung, University of Zurich, for providing the funding.

\section{Author details}

'Biomedical Optics Research Laboratory (BORL), Division of Neonatology, Department of Obstetrics and Gynecology, University Hospital Zurich, Frauenklinikstrasse 10, 8091 Zurich, Switzerland. ${ }^{2}$ Institute of 
Neuroinformatics (INI), University of Zurich and ETH Zurich,

Winterthurerstrasse 190, 8057 Zurich, Switzerland. ${ }^{3}$ Molecular Imaging and

Functional Pharmacology, Institute for Biomedical Engineering, ETH and

University of Zurich, Wolfgang-Pauli-Strasse 27, 8093 Zurich, Switzerland.

Received: 22 April 2010 Accepted: 2 December 2010

Published: 2 December 2010

\section{References}

1. Ertelt $D$, et al: Action observation has a positive impact on rehabilitation of motor deficits after stroke. Neurolmage 2007, 36(Suppl 2):T164-T173.

2. De Vries S, Mulder T: Motor imagery and stroke rehabilitation: a critical discussion. J Rehabil Med 2007, 37(1):5-13.

3. Buccino G, Solodkin A, Small S: Functions of the mirror neuron system: implications for neurorehabilitation. Cogn Behav Neurol 2006, 19(1):55-63.

4. Jeannerod M: The representing brain: Neural correlates of motor intention and imagery. Behav Brain Res 1994, 17:187-245.

5. Rizzolatti $G$, et al: Premotor cortex and the recognition of motor actions. Brain Res Cogn Brain Res 1996, 3(2):131-41

6. Lotze $\mathrm{M}$, et al: Activation of cortical and cerebellar motor areas during executed and imagined hand movements: an fMRI study. J Cogn Neurosci 1999, 11(5):491-501.

7. Rizzolatti $G$, et al: Premotor cortex and the recognition of motor actions. Brain Res Cogn Brain Res 1999, 3(2):131-142.

8. Fadiga $L$, et al: Motor facilitation during action observation: a magnetic stimulation study. J Neurophysiol 1995, 73(6):2608-2611.

9. Eng K, et al: Interactive visuo-motor therapy system for stroke rehabilitation. Med Biol Eng Comput 2007, 45:901-907.

10. Hoshi Y, Tamura M: Detection of dynamic changes in cerebral oxygenation coupled to neuronal function during mental work in men Neuroscience Letters 1993, 150:5-8.

11. Wolf $M$, Ferrari $M$, Quaresima V: Progress of near-infrared spectroscopy and topography for brain and muscle clinical applications. J Biomed Opt 2007, 12(6):062104.

12. Muehlemann $T$, Haensse $D$, Wolf M: Wireless miniaturized in-vivo near infrared imaging. Optics Express 2008, 16(14):10323-30.

13. lacoboni M, et al: Grasping the intentions of others with one's own mirror neuron system. PLOS Biol 2005, 3(3):e79.

14. Filimon $F$, et al: Human cortical representations for reaching: Mirror neurons for execution, observation, and imagery. Neurolmage 2007, 37(4):1315-1328

15. Gazzola V, Keysers C: The Observation and Execution of Actions Share Motor and Somatosensory Voxels in all Tested Subjects: Single-Subject Analyses of Unsmoothed fMRI Data. Cereb Cortex 2009, 19(6):1239-1255.

16. Shimada $S$, Abe R: Modulation of the motor area activity during observation of a competitive game. NeuroReport 2009, 20(11):979-983.

17. Shimada S, Hiraki K: Infant's brain responses to live and televised action. Neuroimage 2006, 32(2):930-939.

18. Shibata H, Suzuki M, Gyoba J: Cortical activity during the recognition of cooperative actions. Cog Neuroscience Neuropsychology 2007, 18(7):697-701.

19. Shiraishi $T$, et al: Observation and imitation of nursing actions: A NIRS study with experts and novices. Stud Health Technol Inform 2006, 122:820-1

20. Fuchino $Y$, et al: High cognitive function of an ALS patient in the totally locked-in state. Neuroscience Letters 2008, 435(2):85-89.

21. Miyai I, et al: Cortical Mapping of Gait in Humans: A Near-Infrared Spectroscopic Topography Study. Neurolmage 2001, 14(5):1186-1192.

22. Cooper $C$, et al: NIRS-detected changes in the motor cortex during mental rehearsal of physical activity (imaginary exercise). Adv Exp Med Biol 2006, 578:185-90.

23. Wriessnegger SC, Kurzmann J, Neuper C: Spatio-temporal differences in brain oxygenation between movement execution and imagery: A multichannel near-infrared spectroscopy study. International Journal of Psychophysiology 2008, 67(1):54-63.

24. Sitaram R, et al: Temporal classification of multichannel near-infrared spectroscopy signals of motor imagery for developing a brain-computer interface. Neurolmage 2007, 34(4):1416-1427.

25. Coyle S, Ward T, Markham C: Brain-computer interface using a simplified functional near-infrared spectroscopy system. J Neural Eng 2007, 4(3):219-26.
26. Coyle $S$, et al: On the suitability of near-infrared (NIR) systems for nextgeneration brain-computer interfaces. Physiol Meas 2004, 25(4):815-22.

27. Kanoh $S$, et al: A NIRS-based brain-computer interface system during motor imagery: System development and online feedback training. Engineering in Medicine and Biology Society EMB, Annual International Conference of the IEEE 2009.

28. Oldfield R: The assessment and analysis of handedness: the Edinburgh inventory. Neuropsychologia 1971, 9(1):97-113.

29. Bricker C: Easy Randomizer, Version 4.1.

30. Colebatch JG, et al: Regional cerebral blood flow during voluntary arm and hand movements in human subjects. J Neurophysiol 1991, 65(6):1392-1401.

31. Pulvermuller $F$, et al: Motor programming in both hemispheres: an EEG study of the human brain. Neuroscience Letters 1995, 190:5-8.

32. Rao SM, et al: Functional magnetic resonance imaging of complex human movements. Neurology 1993, 43(11):2311.

33. Solodkin A, et al: Lateralization of motor circuits and handedness during finger movements. European Journal of Neurology 2001, 8:425-434.

34. Muehlemann $T$, et al: In vivo functional near-infrared spectroscopy measures mood-modulated cerebral responses to a positive emotional stimulus in sheep. Neurolmage 2010, 54(2):1625-1633.

35. Jaspers $\mathrm{H}$ : The ten-twenty electrode system of the International Federation. In: Electroencephalography and clinical neurophysiology. Electroencephalogr Clin Neurophysiol 1958, 10:371-375.

36. Buccino $G$, Riggio $L$ : The role of the mirror neuron system in motor learning. Kinesiology 2006, 38(1):5-15.

37. Delpy $D$, et al: Estimation of optical path length through tissue from direct time of flight measurements. Phys Med Biol 1988, 33:1433-42.

38. Wray $S$, et al: Characterization of the near infrared absorption spectra of cytochrome aa3 and haemoglobin for the non-invasive monitoring of cerebral oxygenation. Biochimica et Biophysica Acta (BBA) - Bioenergetics 1988, 933(1):184-192

39. Zhao $\mathrm{H}$, et al: Maps of optical differential pathlength factor of human adult forehead, somatosensory motor and occipital regions at multiwavelengths in NIR. Phys Med Biol 2002, 47:2075-2093.

40. Strangman $\mathrm{G}$, et al: A quantitative comparison of simultaneous BOLD fMRI and NIRS recordings during functional brain activation. Neurolmage 2002, 17:719-731.

41. Adamovich SV, et al: Sensorimotor training in virtual reality: A review. NeuroRehabilitation 2009, 25(1):29-44.

42. Hoshi Y, Chen S: New Dimension of Cognitive Neuroscience Research with Near-Infrared Spectroscopy: Free-Motion Neuroimaging Studies. In Progress in Brain Mapping Research.. 1 edition. Edited by: Science N. New York; 2006:205-229.

43. Beisteiner $R$, et al: Mental representations of movements. Brain potentials associated with imagination of hand movements. Electroencephalogr Clin Neurophysiol 1995, 96(2):183-93.

44. Grèzes J, Decety J: Functional anatomy of execution, mental simulation, observation, and verb generation of actions: a meta-analysis. Hum Brain Mapp 2001, 12(1):1-19.

45. Holper $\mathrm{L}$, Wolf M: Motor imagery in response to fake feedback measured by functional near-infrared spectroscopy. Neuroimage 2010, 50(1):190-7.

46. Okada E, Delpy D: Near-infrared light propagation in an adult head model. I. Modeling of low-level scattering in the cerebrospinal fluid layer. Appl Opt 2003, 42(16):2906-14.

47. Okada E, Delpy D: Near-infrared light propagation in an adult head model. II. Effect of superficial tissue thickness on the sensitivity of the near-infrared spectroscopy signal. Appl Opt 2003, 42(16):2915-22.

48. Sirigu $A$, et al: The mental representation of hand movements after parietal cortex damage. Science 1996, 273(5281):1564-8.

49. Malouin F, et al: Bilateral slowing of mentally simulated actions after stroke. NeuroReport 2004, 15(8):1349-53.

50. Sabaté M, González B, Rodríquez M: Brain lateralization of motor imagery: motor planning asymmetry as a cause of movement lateralization. Neuropsychologia 2004, 42(8):1041-1049.

51. Ang $\mathrm{K}$, et al: A clinical evaluation on the spatial patterns of non-invasive motor imagery-based brain-computer interface in stroke. Conf Proc IEEE Eng Med Biol Soc 2008.

52. Liang $\mathrm{N}$, et al: Further evidence for excitability changes in human primary motor cortex during ipsilateral voluntary contractions. Neuroscience Letters 2008, 433(2):135-140. 
53. Babiloni F, et al: Brain computer interface: estimation of cortical activity from non invasive high resolution EEG recordings. Conf Proc IEEE Eng Med Biol Soc 2004.

54. Holper L, Biallas M, Wolf M: Task complexity relates to activation of cortical motor areas during uni- and bimanual performance: $\mathrm{A}$ functional NIRS study. Neurolmage 2009, 46(4):1105-1113.

55. Hess CW, Mills KR, Murray NMF: Magnetic stimulation of the human brain: Facilitation of motor responses by voluntary contraction of ipsilateral and contralateral muscles with additional observations on an amputee. Neuroscience Letters 1986, 71(2):235-240.

56. Muellbacher $W$, et al: Changes in motor cortex excitability during ipsilateral hand muscle activation in humans. Clinical Neurophysiology 2000, 111(2):344-349

57. Tinazzi M, Zanette G: Modulation of ipsilateral motor cortex in man during unimanual finger movements of different complexities. Neuroscience Letters 1998, 244(3):121-124.

58. Shibuya K, Kuboyama N: Human motor cortex oxygenation during exhaustive pinching task. Brain Research 2007, 1156:120-124.

59. Cramer SC, et al: Activation of Distinct Motor Cortex Regions During Ipsilateral and Contralateral Finger Movements. J Neurophysiol 1999, 81(1):383-387.

60. Verstynen T, et al: Ipsilateral Motor Cortex Activity During Unimanual Hand Movements Relates to Task Complexity. J Neurophysiol 2005, 93(3):1209-1222

61. Mattay $V$, et al: Hemispheric control of motor function: a whole brain echo planar fMRI study. Psychiatry Res 1998, 83(1):7-22.

62. Singh $L$, et al: Comparison of ipsilateral activation between right and left handers: a functional MR imaging study. [Miscellaneous Article]. Neuroreport 1998, 9(8):1861-6.

63. Porro C, et al: Primary motor and sensory cortex activation during motor performance and motor imagery: a functional magnetic resonance imaging study. J Neurosci 1996, 16(23):7688-7698.

doi:10.1186/1743-0003-7-57

Cite this article as: Holper et al:: Testing the potential of a virtual reality neurorehabilitation system during performance of observation, imagery and imitation of motor actions recorded by wireless functional nearinfrared spectroscopy (fNIRS). Journal of NeuroEngineering and Rehabilitation 2010 7:57.

\section{Submit your next manuscript to BioMed Central and take full advantage of:}

- Convenient online submission

- Thorough peer review

- No space constraints or color figure charges

- Immediate publication on acceptance

- Inclusion in PubMed, CAS, Scopus and Google Scholar

- Research which is freely available for redistribution

Submit your manuscript at www.biomedcentral.com/submit
Biomed Central 